\title{
La formación de traductores en Flandes: la influencia del sector de la traducción en los métodos de enseñanza
}

\section{Translator training in Flanders: the influence of the translation sector on teaching methods}

\section{Guillermo SANZ GALLEGO}

Universiteit Gent

\section{Guillermo.SanzGallego@ugent.be}

Recibido: 13-1-2016. Revisado: 26-10-2016. Aceptado: 1-11-2016.

Resumen: El presente artículo pretende explicar cómo se han trazado y acuñado una serie de directrices metodológicas innovadoras en el Departamento de Traducción, Interpretación y Comunicación Multilingüe de la Universidad de Gante con el objetivo de optimizar la formación académica de los futuros profesionales del sector, aportándoles los conocimientos teóricos que exige el mercado laboral, así como dotándolos de una sólida base práctica. Las conclusiones que sirvieron de base para trazar estas directrices metodológicas fueron fruto de una encuesta y una serie de mesas redondas organizadas en 2013 entre un grupo de profesores de asignaturas de traducción de la Universidad de Gante y un grupo de profesionales y especialistas de los sectores de la traducción, la interpretación y la comunicación de Flandes, tales como directores de agencias flamencas de traducción y coordinadores de secciones o de proyectos de 
organismos oficiales belgas. El artículo muestra cómo el perfil de traductor al que alude este grupo de trabajo difiere del que se ha tomado como referente tradicionalmente: el modelo institucional diseñado por el EMT Expert Group. Los resultados y conclusiones que se exponen a continuación pueden arrojar luz no solo sobre la política de traducción adoptada en Flandes, sino también sobre la relación entre una de las lenguas minoritarias de la UE, como es el neerlandés, y uno de los idiomas más hablados del mundo, el español.

Palabras clave: Estudios de Traducción; formación de traductores; métodos de enseñanza; recursos didácticos; neerlandés; traducción inversa.

\begin{abstract}
This article aims at clarifying how a series of innovative methodological guidelines have been outlined and implemented within the Department of Translation, Interpreting and Communication at Ghent University for the purposes of optimizing academic education of future professionals of the sector, as well as providing them with both the theoretical background demanded by the labour market and a thorough training. Such conclusions were the result of a survey and a number of roundtables organized in 2013 by a group of lecturers of Translation Studies at Ghent University and several experts and professionals of the translation sector in Flanders. The latter group included heads of Flemish translation agencies, as well as department coordinators of Belgian official organizations. The article points out how the translator profile alluded to by the working group differs from the institutional model designed by the EMT Expert Group, which has traditionally been taken as the main reference. The results and conclusions reported below can shed light not only on the translation policy adopted in Belgium, but also on the relationship between one of the minority languages of the EU, such as Dutch, and one of the most spoken languages in the world, such as Spanish.
\end{abstract}

Keywords: Translation Studies; translator training; teaching methods; didactic resources; Dutch; inverse translation.

\title{
1. INTRODUCCIÓN
}

El presente artículo pretende subrayar una serie de elementos esenciales en la formación de futuros traductores e intérpretes flamencos. Este listado fue consensuado en el marco de un acuerdo de cooperación de un grupo de trabajo llamado Resonansgroep Master in het Vertalen y formado por un grupo de profesores del Departamento de Traducción, Interpretación y Comunicación Multilingüe de la Universidad de Gante y una serie de profesionales del sector de la traducción en Flandes.

Llama la atención cómo las competencias mencionadas por este grupo de trabajo trazan el perfil de un traductor determinado que no coincide con el que diseñan otros grupos de trabajo como el del EMT Expert Group. Mientras que el Resonansgroep

Guillermo SANZ GALLEGO

La formación de traductores en Flandes: la influencia del sector de la traducción en los métodos de enseñanza 
Master in het Vertalen se centra en las competencias y el tipo de encargo al que se enfrentan los profesionales que trabajan en el sector de la traducción en Flandes, es decir, principalmente como autónomos o en plantilla para agencias o para empresas, el EMT Expert Group tiene como referencia un perfil más institucional como puede verse en su página web, donde el hipervínculo que aparece remite a la web de la Dirección General de Traducción de la Comisión Europea y, en concreto, al perfil de sus traductores.

Otra diferencia destacable es que mientras que en el Resonansgroep se han ordenado las diferentes competencias por orden de relevancia de acuerdo con una encuesta, el EMT Expert Group no ha llevado a cabo esa clasificación y únicamente menciona un listado de aptitudes. Precisamente esta ha sido una de las críticas que ha recibido el informe del EMT Expert Group desde el año de su publicación, en 2009, en concreto por parte de Lafeber $(2012,110)$, quien destaca que el listado carece de matices en lo que se refiere a la importancia relativa de las competencias. También han surgido voces críticas con el concepto de competencia adoptado en el informe. Pym (2013, 490), por ejemplo, argumenta con razón que en muchas ocasiones el informe del EMT Expert Group no trata de competencias, es decir, "saber cómo", sino de meros conocimientos, o simplemente «ser consciente de algo".

Como se ha mencionado anteriormente, el Departamento de Traducción, Interpretación y Comunicación Multilingüe de la Universidad de Gante inició en 2013 una serie de contactos con un grupo de profesionales del sector de traducción en Flandes con intención de mejorar la formación de los futuros traductores e intérpretes flamencos. Aquellos contactos tenían como principal objetivo trazar el perfil del traductor que buscan las empresas del sector establecidas en Flandes a partir de las competencias más valoradas. Estos encuentros se materializaron en una encuesta y en una serie de mesas redondas que fueron especialmente enriquecedoras para adoptar metodologías innovadoras en las asignaturas prácticas de traducción. El grupo de profesionales estaba formado por 10 representantes del sector de la traducción, 3 del sector de la interpretación y 4 del sector de la comunicación. Sus funciones y puestos eran variados, encontrándose entre ellos directores de agencias de traducción, coordinadores de secciones o de proyectos de organismos oficiales, directivos y empleados de empresas dedicadas a la comunicación. Los resultados de la encuesta se encuentran más abajo en el anexo.

Las conversaciones que se mantuvieron en este grupo de trabajo iban destinadas a dar respuesta a cuáles deben ser las prioridades en la formación de futuros traductores, qué base teórica deben tener los estudiantes y a qué tipos de ejercicios se deben dedicar las clases. Llama la atención cómo en general suele haber consenso entre los integrantes del grupo de trabajo. Por orden de importancia, las cuestiones prioritarias de la carrera deberían ser en primer lugar la orientación hacia el mercado laboral, seguida de un refuerzo del bagaje cultural, en tercer lugar mejorar y afianzar 
el nivel de neerlandés, seguido del perfeccionamiento del conocimiento de idiomas extranjeros, a continuación la capacidad de análisis textual junto con el conocimiento de diferentes estrategias de traducción, las competencias sociales, especializarse en un determinado campo y, por último, el conocimiento de herramientas informáticas.

Más adelante se detallan las conclusiones de aquellos encuentros y cuáles fueron las repercusiones en el programa de asignaturas de traducción dentro de la sección de español y, en concreto, en las asignaturas de traducción inversa, es decir, traducción del neerlandés al español, tanto en segundo como en cuarto. En una serie de casos se afianzaron iniciativas con las que ya se venía trabajando desde hacía algún tiempo; en otros casos se implantaron nuevas fórmulas o se terminó por descartar otras. Asimismo es importante recalcar que en todo momento se tuvo muy presente la idiosincrasia del sector de la traducción en Flandes y que, como se observará más adelante, una serie de prioridades que se detallan son características de este mercado en concreto. Lógicamente, este listado de competencias completa y matiza el del EMT que, como ya se ha indicado, se centra principalmente en los traductores de las instituciones europeas.

\section{COMPETENCIAS PRIORITARIAS PARA LOS EXPERTOS DEL SECTOR $^{1}$}

\subsection{Orientación al mercado laboral}

La orientación hacia el mercado laboral de los futuros profesionales del sector es sin duda la mayor prioridad para los especialistas consultados. En este sentido destaca la importancia de la gestión del tiempo a lo largo de las diferentes fases del proceso traductivo (lectura preliminar, análisis del texto y del perfil del lector meta, consulta de fuentes, traducción y revisión), así como la gestión de proyectos, es decir, hacer ver al grupo de estudiantes que es esencial respetar las fechas de entrega de cualquier encargo, incluso en situaciones de estrés. Otro elemento que demandan los especialistas es proporcionar un marco adecuado en las clases para que se realicen actividades que puedan entenderse como simulaciones del tipo de trabajo que se realiza en la vida real, tanto traducciones como revisiones.

1. Para cada uno de los aspectos comentados en el punto 2., se puede consultar el gráfico correspondiente en el Anexo. 


\subsection{Bagaje cultural}

El bagaje cultural de los futuros profesionales es otra de las principales demandas del sector. El candidato ideal debe mostrar conocimientos de cultura general y, en concreto, ser un experto en la cultura tanto de la lengua fuente como de la lengua meta. Los especialistas del sector destacan una serie de campos, como el político, económico, social, histórico, literario, artístico y cinematográfico, y añaden que todo ello repercute tanto en el ritmo de trabajo como en la garantía de calidad de las traducciones. Además, se espera de los estudiantes que al finalizar su carrera académica sean capaces de desarrollar una rutina de traducción y una serie de recursos que les permitan proponer soluciones convincentes para la traducción de las referencias culturales, dependiendo de la tipología del texto, su función en la cultura meta y las características preestablecidas del lector final.

Para ello es necesario dedicar también atención a diferentes métodos de traducción que ayuden a crear un texto meta más domesticado o extranjerizado según las necesidades. Asimismo es conveniente proporcionar un marco adecuado para desarrollar y afianzar estrategias heurísticas que además de hacer énfasis en el plano semántico hagan hincapié en la cultura, incorporando a la fase de documentación un amplio abanico de fuentes que complementen la consulta de diccionarios, como por ejemplo las enciclopedias, las bases de datos, las hemerotecas y los textos paralelos. Finalmente se destaca que, además de disponer de un bagaje cultural que les permita desenvolverse con garantías en la traducción de textos con un nivel de dificultad considerable, es conveniente que durante su formación los futuros traductores vayan siendo conscientes de la importancia de ampliar eses tipo de conocimientos.

\subsection{Neerlandés}

El dominio del neerlandés es otro de los aspectos esenciales en los que insisten los especialistas consultados. No es ninguna novedad que desde el sector se exija un excelente nivel de la lengua meta a los futuros traductores. Les piden que posean los recursos adecuados para expresar ideas complejas, matices o cambios de registro, y que sean capaces de hacer un uso adecuado de los sinónimos y los signos de puntuación.

Lo que sí es característico del mercado laboral flamenco es que se exige un conocimiento diferenciado de las variedades lingüísticas del neerlandés de Flandes y del de los Países Bajos. Ello viene condicionado por una demanda cada vez mayor de un producto más localizado, es decir, adaptado a una cultura lingüística determinada. Los responsables del sector de la traducción consultados coinciden en que durante la formación de los futuros profesionales se les debe sensibilizar para que le presten la máxima atención a este requisito, ya que en el sector es indispensable producir textos 
tanto en neerlandés estándar como en cualquiera de las diferentes variedades, aunque lógicamente el mercado flamenco suele demandar en primer lugar textos adaptados a los lectores flamencos.

Por último, es necesario comentar los resultados que aparecen en el anexo sobre este apartado, y muy en especial las respuestas a si se considera prioritario que los estudiantes traduzcan únicamente al neerlandés. Un $8 \%$ de los encuestados lo considera «innecesario" y un 33\% lo ve como algo «secundario", lo cual no debe entenderse, sin embargo, como un desinterés por parte de algunos representantes del sector hacia el neerlandés.

\subsection{Lenguas extranjeras}

La siguiente prioridad es quizá poco común en el sector en general, pero según los expertos consultados se trata de una situación que se da en Flandes. Teniendo en cuenta las características específicas del mercado neerlandófono, en términos de volumen y de número de traductores, los especialistas del sector destacan la importancia de las lenguas extranjeras, ya que no es extraño que en este mercado se realicen traducciones inversas. Incluso puede ocurrir que un traductor neerlandófono lleve a cabo la revisión de un texto en otro idioma, como el español, aunque esta opción es poco frecuente y solo se considera en caso de no encontrar ningún nativo disponible para el encargo en cuestión. En este sentido, los especialistas del sector opinan que es indispensable que los futuros traductores sean conscientes de que a lo largo de su carrera no van a estar expuestos únicamente a encargos de traducción directa y que, por eso, deben estar preparados para realizar eventuales encargos de traducción inversa. La comunicación en otros idiomas también se estima prioritaria de cara a poder adaptarse a las tareas cotidianas del mercado laboral flamenco, aunque cabe matizar que en el sector de la traducción se le da preferencia a la comunicación escrita, es decir, ser capaz de escribir una carta o un mensaje de correo electrónico sin errores. La competencia oral se valora positivamente, pero no se considera esencial para poder desempeñar con éxito las funciones de traductor.

\subsection{Análisis de texto y estrategias de traducción}

Otra de las prioridades del sector pasa por dotar al estudiante de los recursos adecuados para llevar a cabo un análisis correcto del texto origen. En primer lugar, se debe conseguir afianzar una rutina de trabajo que le permita identificar la tipología del texto, su estructura, sus características estilísticas, el registro utilizado por el autor, detectar referencias culturales implícitas, pasajes con material políticamente sensible 
en los que la intervención del traductor pueda ser polémica, así como ser capaz de reconocer matices irónicos. Esta fase inicial debe permitir al estudiante trazar el perfil del lector meta, buscar problemas de traducción, razonar si se va a optar por una metodología más extranjerizadora o domesticadora, así como determinar qué estrategias se van a ir adoptando en cada fragmento. Algunos especialistas consideran que se debe concienciar a los estudiantes de la importancia de este análisis y creen que debe enmarcarse dentro de la fase de gestión del proyecto.

\subsection{Competencias sociales}

Las competencias sociales ocupan el siguiente lugar en cuanto a valoración por parte de los especialistas del sector. A este respecto destacan la autonomía a la hora de analizar y resolver problemas, el interés por aprender, ser dinámico, la rapidez y facilidad de adaptación a nuevas situaciones, así como ser capaz de comunicarse de forma fluida y eficaz tanto con compañeros como con agencias y clientes.

Los representantes del sector también subrayan la necesidad de reforzar la autoconfianza de los futuros traductores, ya que en general la percepción que tienen de los recién licenciados flamencos es que suelen carecer de la autoestima suficiente para defender con asertividad sus versiones y opiniones. Además, se observa cierta hipersensibilidad a la hora de aceptar críticas, lo cual, unido a una falta de confianza en sí mismo, puede incidir negativamente tanto en el profesional como en el ambiente laboral y en la colaboración en equipo.

\subsection{Especialización}

La especialización del traductor es una característica que en el mercado flamenco se valora positivamente, aunque no en un primer momento, sino a partir de una determinada fase de la vida laboral. Es lógico que las empresas no exijan que los recién licenciados posean profundos conocimientos de alguna materia en concreto o que sean especialistas en campos como el jurídico, científico-técnico, económico o médico, entre otros, ya que las agencias suelen confiar este tipo de encargo a traductores con mayor experiencia. Sin embargo, lo que sí se agradece en el sector es que los jóvenes licenciados sean capaces de encontrar terminología especializada. Desde el sector se advierte también de la poca frecuencia con la que se realizan encargos de tipo periodístico, dado que en Flandes suelen ser los propios periodistas, si no las agencias de noticias o los periódicos, los que se encargan de traducir y adaptar artículos o noticias. Los representantes del sector tampoco ven muy necesario que los estudiantes se enfrenten a textos literarios, teniendo en cuenta las características de 
este tipo de encargo, poco demandado por las agencias y habitualmente realizado por traductores con un perfil muy distinto.

\subsection{Herramientas informáticas}

Los especialistas coinciden en que el conocimiento de herramientas informáticas y de software especializado debe ser también una prioridad en la formación académica de los futuros traductores, aunque en general destacan que no es algo imprescindible, ya que se trata de conocimientos que pueden adquirirse una vez iniciada la carrera profesional. En concreto, se menciona que lo que más valoran las empresas es que los futuros profesionales demuestren flexibilidad y sean capaces de adaptarse al software, a las memorias de traducción o a las herramientas que utilice la empresa. En cualquier caso, de entre todas las herramientas, los expertos destacan la importancia de SDL Trados Studio, Wordfast y Multiterm. Llama la atención cómo desde el sector se puede llegar a valorar que un candidato haya tenido experiencia con algún sistema de traducción automático, aunque en general estas competencias no se ven como algo indispensable en la contratación de traductores. También se añade que sí es absolutamente imprescindible tener un buen conocimiento de paquetes de ofimática y, en concreto, procesadores de texto (Word), programas de correo electrónico (Outlook), tablas de cálculo (Excel) y presentaciones de diapositivas (PowerPoint).

\subsection{Otras competencias}

Por último, cabe mencionar una serie de cualidades que valoran positivamente algunos de los expertos consultados. Como aquí no se puede hablar de consenso, estas aptitudes deberían situarse en un segundo plano. Son cuatro: ser capaz de traducir textos de mala calidad, es decir, mal redactados, con faltas de ortografía, imprecisiones, omisiones, errores y erratas; la capacidad de adaptación a cualquier formato de documento; el interés por la actualidad política, económica y social de las culturas en las que se especializa el traductor; y conocer los entresijos del mercado, es decir, ser capaz de establecer el precio de un encargo o saber a quién acudir para solicitar cualquier tipo de ayuda.

\subsection{Evaluación}

Otro de los temas de debate del grupo de trabajo fue la forma de evaluar. Tanto los profesores como los especialistas del sector coincidieron en que los errores de 
interpretación deben sancionarse con más dureza, aunque ciertos errores graves de expresión también deben llevarse a ese mismo nivel de gravedad. Se mencionó, además, la necesidad de familiarizar a los alumnos con la forma de evaluar de algunas instituciones y agencias donde únicamente existen dos calificaciones: apto o no apto. Es conveniente que el estudiante sea consciente de que un ejercicio o prueba de traducción se evalúa como apto únicamente si el cliente puede utilizarlo inmediatamente sin necesidad de pasar por un revisor, es decir, si la traducción está impecable, mientras que la calificación de no apto implica que la traducción contiene errores.

\section{INICIATIVAS DEL DEPARTAMENTO PARA FOMENTAR LA IMPLEMENTACIÓN DE LAS COMPETENCIAS CONSIDERADAS PRIORITARIAS POR EL SECTOR FLAMENCO DE LA TRADUCCIÓN}

Una vez enumeradas las competencias mejor valoradas por los representantes del mercado pasamos a explicar cómo han influido estas mesas redondas y encuestas en la programación de asignaturas de traducción neerlandés-español, tanto en segundo curso como en cuarto. En general se han tenido en cuenta todos los comentarios y se han seguido todas las indicaciones de los expertos del sector a la hora de impartir estas asignaturas.

La prioridad principal, que era la orientación hacia el mercado laboral, ha sido determinante a la hora de establecer un marco de trabajo, es decir, se ha intentado que las actividades realizadas en clase reflejen con el mayor realismo posible las situaciones cotidianas a las que se enfrentan los traductores. Además, se ha intentado alternar diferentes métodos de trabajo para fomentar una mayor flexibilidad de los alumnos. Por esa razón se ha considerado conveniente dedicar atención a ejercicios realistas de traducción en grupo, es decir, traducir textos más extensos que los que se utilizan para encargos individuales o para exámenes con la intención de que sea necesario segmentarlos y repartir el trabajo entre varios traductores para finalmente realizar la labor de revisión en grupo antes de entregar la traducción consensuada. Como se ha dicho anteriormente, es recomendable también alternar este tipo de actividad con otras que permitan reflexionar sobre otros aspectos con más detalle, ya que desde el sector se demanda también que los futuros traductores demuestren cierta flexibilidad a la hora de adaptarse a diferentes formas de trabajo o metodologías dependiendo de los clientes o de las circunstancias laborales. Un buen ejemplo puede ser realizar seminarios de traducción en los que se comparen diversas versiones, se identifiquen errores y se propongan correcciones o alternativas. Otra fórmula puede implicar la elaboración de una traducción consensuada por toda la clase, es decir, tras 
la fase de análisis del texto origen, los alumnos van dictando sus traducciones de cada segmento, para posteriormente someterlos a aprobación en grupo. También se puede optar por presentaciones individuales o por parejas de estudiantes que defiendan sus traducciones razonando sus decisiones.

Otro tipo de actividad orientada a reflejar de forma realista el mercado laboral tiene que ver con la revisión de traducciones. En efecto, como se apuntaba anteriormente, la revisión debe convertirse en una fase esencial del proceso de traducción. A este respecto conviene poner énfasis en diferentes aspectos, prácticas y procedimientos tratados por autores de referencia en este campo como Payne (1987), Lorenzo (2002), Pavlovic y Antunovic (2011) y Mossop (2014). Afortunadamente, durante varios cursos académicos la asignatura de Traducción General NL-ES se ha beneficiado en el Departamento de Traducción de la Universidad de Gante de la programación simultánea de otra asignatura, Translation EN-ES, organizada principalmente para estudiantes españoles del programa de intercambio Erasmus. La coincidencia de estas dos asignaturas en el mismo cuatrimestre ha permitido organizar actividades comunes muy enriquecedoras para ambos grupos, como las revisiones realizadas por los estudiantes del programa de intercambio Erasmus a las traducciones de los estudiantes neerlandófonos. En estas actividades se evalúa a los estudiantes nativos españoles a partir de las correcciones que realizan, teniendo en cuenta diferentes criterios como la identificación de errores o el uso de fuentes para razonar las correcciones. Durante varios cursos académicos, los estudiantes del Departamento de Traducción se han podido beneficiar de esta cooperación tan fructífera y de la simulación realista de un encargo para una agencia de traducción. Sin embargo, a partir del curso 2015-2016 se produce una reforma en el programa de la carrera de traducción y la asignatura de Traducción General NL-ES pasa a impartirse en el segundo cuatrimestre, lo cual impide organizar actividades conjuntas por la dificultad que supone hacer coincidir ambos grupos. Por consiguiente, los ejercicios de revisión se realizan a partir de entonces entre compañeros de la misma asignatura, lo cual también es enriquecedor.

Para reforzar el bagaje cultural se ha optado por seleccionar textos de carácter general que contengan referencias culturales y que presenten algún tipo de desafío para los futuros traductores. En este sentido destacan los textos sacados de folletos turísticos, textos publicitarios, reseñas de libros, prólogos, biografías, o agradecimientos, aunque también pueden suponer cierta dificultad en ese aspecto los discursos o informes anuales de una empresa. En este tipo de encargo es esencial que los estudiantes lleven a cabo una rigurosa fase de documentación para reconocer las referencias a determinados personajes, topónimos, instituciones, funciones o cargos, expresiones, refranes y episodios históricos, por dar algunos ejemplos que mencionan autores como Snell-Hornby (1995), Aixelá (1996), Leppihalme (1997) y Beller y Leerssen (2007). En general, los estudiantes neerlandófonos demuestran un

Guillermo SANZ GALLEGO

La formación de traductores en Flandes: la influencia del sector de la traducción en los métodos de enseñanza
CLINA

vol. 2-2, December 2016, 71-90

elSSN: 2444-1961

(C) Ediciones Universidad de Salamanca - CC BY-NC-ND 
gran rigor a la hora de buscar soluciones a este tipo de desafío, a pesar de la dificultad añadida que entraña enfrentarse a un ejercicio de traducción inversa. Sin embargo, suelen encontrar más problemas a la hora de proporcionar soluciones para referencias culturales implícitas, como, por poner un ejemplo, la traducción de tres colores, «rood, oranje, en groen», que traducen palabra por palabra como «rojo, naranja y verde», sin pararse a pensar que el texto hace una clara referencia a un semáforo y que en España los colores oficiales de esta señal luminosa son «rojo, ámbar y verde».

Respecto al desafío que plantea la traducción inversa para los estudiantes neerlandófonos habría que señalar algunos detalles. En primer lugar, llama la atención la escasa aparición de errores graves de interpretación del texto origen, como pueden ser los contrasentidos, más si cabe si se compara el grupo de estudiantes de esta asignatura con el de los estudiantes españoles de intercambio del programa Erasmus que tienen que traducir del inglés a su lengua materna. Esto puede deberse a que, en el caso de la traducción inversa, el texto original está escrito en la lengua materna del traductor, lo que supone una ventaja en lo que se refiere a la comprensión del texto origen si se compara con una actividad de traducción directa en la que el texto origen aparece en un idioma distinto a la lengua materna. Es necesario matizar que la menor frecuencia de aparición de errores de interpretación no significa que no se den casos en los que el sentido del texto meta se separe del texto origen. A continuación se detallan los errores más frecuentes de los estudiantes neerlandófonos al realizar traducciones inversas.

En cuanto a errores de expresión deberíamos destacar el uso de los tiempos del pasado y el subjuntivo, la conjugación de los verbos, la concordancia en los sintagmas nominales, el uso de las preposiciones, los pronombres, los relativos, las confusiones entre adjetivos y adverbios como en la traducción de «goed» (es decir, uso indistinto de «buen(o)» y «bien»), las construcciones impersonales, el uso de la voz pasiva, las construcciones con "gustar» e «interesar», la ausencia de perífrasis verbales y ciertos errores ortográficos como los acentos.

El uso del léxico merece una mención aparte, ya que en general se observan numerosas dificultades, como los errores causados por los falsos amigos o la aparición de muestras de ambigüedad en el texto meta en casos en los que no la hay en el texto origen. También suele observarse una falta de análisis componencial en situaciones como en la traducción de «heilig» o «wild», que dependiendo del sustantivo al que se aplique será «santo» (para personas) o «sagrado» (para animales o cosas) en el primer caso y «salvaje» (para personas y animales) o «silvestre» (para plantas) en el segundo. Casos similares se dan en la traducción de conjunciones como «maar» o «als», ya que se suelen confundir «pero» con «sino», y «si» con «cuando». En general, los estudiantes neerlandófonos pecan de precavidos y suelen optar por generalizaciones en lugar de arriesgarse con fórmulas más específicas, por lo que no es extraño encontrarse en alguna traducción un adjetivo con un sentido amplio, como «raro», como traducción de 
"eigenaardig" en casos en los que se desee enfatizar y alabar el carácter extraordinario de un fenómeno, algo que habría que expresar con otras opciones más concretas, como "peculiar», "Curioso", "singular» u "original». No obstante, también es cierto que en alguna ocasión se dan casos muy distintos, como la traducción en un texto académico de "citaties en notities» como "citaciones y noticias", mientras que lo adecuado sería "citas y notas". Las colocaciones también suelen ser problemáticas, ya que a no pocos estudiantes les cuesta trabajo dejar de traducir palabra por palabra en expresiones como "een afspraak maken» o "een fout maken», donde en lugar de "concertar una cita» o "cometer un error» suelen decantarse por el verbo «hacer» («maken»). Lo mismo ocurre con la expresión «een boete geven», habitualmente expresada por muchos de los estudiantes como «dar una multa» en lugar de «poner una multa».

Por último, sería conveniente mencionar otros aspectos a tener en cuenta respecto a las dificultades que encuentran los estudiantes neerlandófonos al realizar una traducción inversa al español. Las diferencias entre ambos idiomas hacen que en general les resulte más complicado el trasvase de algunas construcciones, como aquellas en las que aparecen enumeraciones de adjetivos en neerlandés. En este tipo de caso es en el que se puede apreciar qué estudiantes son capaces de aplicar estrategias de traducción que les permitan proporcionar soluciones más idiomáticas en español. Nos referimos en concreto a estrategias tales como transposiciones (o cambios de categoría gramatical, es decir, sustituir alguno de los adjetivos por un adverbio, un sustantivo o un verbo, por ejemplo), modulaciones (o cambios de perspectiva), o compensaciones (la pérdida de significado en un segmento que se compensa en otro). Asimismo suele observarse en general un uso excesivo del verbo «recibir» («krijgen») en construcciones en las que el español tiende a optar por cambios de perspectiva, como en «ik heb een boek gekregen", traducido como "he recibido un libro" en vez de «me han regalado un libro». Otra expresión que los estudiantes suelen utilizar en exceso es «un poco» en casos en los que en español no se usa, como en la traducción de «het is een beetje zoals...»; antes que «es un poco como...» se le daría preferencia a cambiar de verbo, como en «se parece a...». Este tipo de error suele también observarse con frecuencia en la gradación incorrecta de adjetivos y adverbios elativos, como «un poco horroroso" o «muy excelente». Los adverbios terminados en -mente también suelen aparecer de forma excesiva, en contra de los consejos de los libros de estilo, por ejemplo en "conscientemente o inconscientemente» como traducción de «bewust of onbewust", en lugar de "consciente o inconscientemente». Otro caso parecido de uso incorrecto de los adjetivos es la traducción de «zijn echtgenote, de Duitse...» como «su mujer alemana...» en vez de «su mujer, la alemana...», dando a entender en la traducción que se está informando de un caso de poligamia donde realmente no lo hay.

En cuanto a la siguiente competencia, el perfeccionamiento del neerlandés y de los idiomas extranjeros, en las asignaturas que mencionamos se observa que la mayor

Guillermo SANZ GALLEGO

La formación de traductores en Flandes: la influencia del sector de la traducción en los métodos de enseñanza
CLINA

vol. 2-2, December 2016, 71-90

elSSN: 2444-1961

(C) Ediciones Universidad de Salamanca - CC BY-NC-ND 
dificultad se encuentra en la producción de un texto en una lengua extranjera, mientras que con la lengua materna se trabaja la comprensión escrita, es decir, el conocimiento pasivo del idioma. Como se ha comentado anteriormente, los especialistas del sector valoran de forma muy positiva la oferta de esta asignatura, ya que no es extraño que a lo largo de su carrera los estudiantes neerlandófonos tengan que enfrentarse a encargos de traducción inversa.

Otra de las competencias que demandan los expertos del sector es la capacidad de análisis textual junto con el conocimiento de diferentes estrategias de traducción. Una de las obras de referencia que se recomiendan para que los estudiantes neerlandófonos en general practiquen el análisis de texto es Vertalen wat er staat de Langeveld (1986). También, en cuanto a estrategias de traducción se incorporan taxonomías relevantes de autores como Newmark (1992), Zaro y Truman (1998) y Pym (2010). Es pertinente señalar que en este caso se ha afianzado una rutina con la que ya se llevaba trabajando varios años: al principio de cada clase se realiza un análisis del texto con el que se va a trabajar. Asimismo, en cada fragmento se discuten las diferentes estrategias de traducción adoptadas por cada alumno a la hora de proponer sus versiones. Además, en cada clase se hace hincapié en la necesidad de incorporar la fase de revisión a la rutina de trabajo de cada estudiante para evitar errores que se hayan podido escapar en el proceso de traducción. En esa fase de revisión, que ya se ha mencionado anteriormente, se subraya la importancia de centrarse en primer lugar en una comparativa intertextual entre texto origen y texto meta para comprobar que el mensaje se ha interpretado correctamente, sin omisiones, y en segundo lugar se destaca la importancia de llevar a cabo una revisión de estilo del texto meta.

Las competencias sociales son un aspecto que se intenta fomentar, aunque resulta complicado conseguir un marco óptimo para ello en una clase de traducción, ya que suele tratarse de un grupo numeroso y heterogéneo en el que tampoco se dispone de tiempo suficiente para que en cada sesión cada estudiante tenga la ocasión de razonar sus decisiones. Sí que es cierto que una parte considerable del grupo tiene oportunidad de exponer sus versiones, proponer correcciones o alternativas. También es verdad que, al exponer sus puntos de vista y defender sus traducciones, esos estudiantes van adquiriendo cada vez más confianza en sí mismos.

Por último, es pertinente señalar que otras competencias que mencionan los especialistas a un nivel inferior no se consideran como prioritarias en las asignaturas de traducción general. Nos referimos en concreto a la especialización en un campo determinado y al conocimiento de herramientas informáticas. En cualquier caso, en el currículum académico de este departamento se ofertan varias asignaturas tanto de traducción especializada como sobre el manejo de herramientas, entre ellas localización y terminología. 


\section{CONCLUSIONES}

A modo de conclusión podemos recalcar que las características específicas del mercado laboral flamenco son determinantes a la hora de trazar el perfil ideal de un traductor que, como se ha podido observar, no coincide necesariamente con el modelo institucional presentado por otros grupos de trabajo como el del EMT. Todo esto debe ser tenido en cuenta en la formación académica de los futuros profesionales y por ello es esencial que los departamentos de traducción mantengan un contacto fluido con grupos de representantes del sector para, de este modo, prestar también atención a las demandas y expectativas que puedan distanciarse de las que se establecen desde ciertas instituciones. La comunicación y cooperación entre, por una parte, profesores o formadores y, por otra, empresarios, agencias y autónomos repercute de forma positiva en la preparación de los jóvenes traductores. Además, es necesario tener presente que el sector de la traducción depende enormemente de los pares de lenguas y que el mercado neerlandófono, y el flamenco en concreto, tienen unas características propias que hacen conveniente trazar un perfil muy definido de los futuros traductores.

Se puede afirmar que la cooperación entre los integrantes del grupo de trabajo Resonansgroep Master in het Vertalen en 2013 ha sido altamente productiva para trazar un perfil claro del mercado laboral del sector de la traducción en Flandes, así como para identificar cuáles deben ser las necesidades y prioridades en las que centrar los esfuerzos a la hora de impartir clases de traducción y de reformar el currículum académico de la carrera de traducción que se imparte en el Departamento de Traducción, Interpretación y Comunicación Multilingüe de la Universidad de Gante.

Finalmente, cabe destacar que las conclusiones de este grupo de trabajo sirven también para tener una idea clara de la relación entre uno de los idiomas minoritarios europeos, el neerlandés, y una de las lenguas más habladas del mundo, el español. En el presente artículo se ha podido observar claramente cómo los centros oficiales de formación de traductores flamencos, y en concreto el mencionado departamento de la Universidad de Gante, son capaces de coordinar esfuerzos con los especialistas del sector para buscar fórmulas que fomenten la relación entre el mundo neerlandófono y el hispanohablante, impulsando iniciativas que promuevan el acceso a la información así como el intercambio entre ambas culturas. Quizá la más destacable de estas iniciativas pueda ser la incorporación de asignaturas de traducción inversa del neerlandés al español, algo indispensable en un mercado en el que los futuros traductores son conscientes, desde el momento de su formación, de que a lo largo de su carrera tendrán que enfrentarse en alguna ocasión a la complicada tarea de realizar un traducción inversa o incluso de revisar un texto en otro idioma distinto al suyo. 


\section{REFERENCIAS BIBLIOGRÁFICAS}

AIXELÁ, Javier Franco. 1996. "Culture Specific Items in Translation». En Translation, Power, Subversion, ed. por Román Álvarez y África Vidal. Clevedon: Multilingual Matters, 52-78.

BELLER, Manfred y Joep LEERSSEN. 2007. Imagology - The Cultural Construction and Literary Representation of National Characters: A Critical Survey. Amsterdam: Rodopi.

EMT EXPERT GROUP. 2009. Competences for Professional Translators, Experts in Multilingual and Multimedia Communication. Fecha de acceso 16 de noviembre de 2015. http://ec.europa. eu/dgs/translation/programmes/emt/key_documents/emt_competences_translators_ en.pdf.

EMT EXPERT GROUP WEBSITE. Fecha de acceso 16 de noviembre de 2015. http://ec.europa.eu/ dgs/translation/programmes/emt/index_en.htm.

EUROPEAN COMMISSION WEBSITE (Translator profile for DGT). Fecha de acceso 16 de noviembre de 2015. http://ec.europa.eu/dgs/translation/workwithus/staff/profile/index_en.htm.

LAFEBER, Anne. 2012. "Translation Skills and Knowledge: Preliminary Findings of a Survey of Translators and Revisers Working at Inter-governmental Organizations». Meta 57 (1): 108131.

LANGEVELD, Arthur. 1986. Vertalen wat er staat. Amsterdam/Antwerpen: Atlas Contact.

LEPPIHALME, Ritva. 1997. Culture Bumps: An Empirical Approach to the Translation of Allusions. Clevedon: Multilingual Matters.

LORENZO, María Pilar. 2002. "Competencia revisora y traducción inversa». Cadernos de Traduçao 10: 133-166.

MossoP, Brian. 2014. Revising and Editing for Translators. London/New York: Routledge.

NEWMARK, Peter. 1992. Manual de traducción. [orig. A Textbook of Translation]. Traducido por Virgilio Moya. Madrid: Cátedra.

PAVLOVIC, Nataša y Goranka ANTUNOVIC. 2011. "Here and Now: Self-revision in Student Translation Processes from L2 and L3». Across Languages and Cultures 12 (2): 213-234.

PAYNE, Jerry. 1987. "Revision as a Teaching Method on Translation Courses». En Translation in the Modern Languages Degree, ed. por Hugh Keith e lan Mason. London: Centre for Information on Language Teaching and Research, 43-51.

PYM, Anthony. 2010. Exploring Translation Theories. London/New York: Routledge.

PYM, Anthony. 2013. «Translation Skill-Sets in a Machine-Translation Age». Meta 58 (3): 487 503.

SNELL-HORNBY, Mary, Zuzana JeTtMAROVA y Klaus KAINDL. 1995. Translation as Intercultural Communication. Amsterdam/Philadelphia: John Benjamins.

ZARO, Juan Jesús y Michael TRUMAN. 1998. Manual de Traducción: A Manual of Translation. Alcobendas: SGEL.

Guillermo SANZ GALLEGO

La formación de traductores en Flandes: la influencia del sector de la traducción en los métodos de enseñanza vol. 2-2, December 2016, 71-90

elSSN: 2444-1961

(c) Ediciones Universidad de Salamanca - CC BY-NC-ND 


\section{ANEXO: RESULTADOS DE LA ENCUESTA DEL RESONANSGROEP}

A continuación se presentan los resultados de la encuesta sobre competencias relacionadas con los aspectos comentados. En el caso del tercer gráfico, "Neerlandés", es preciso matizar que los resultados ofrecidos hacen referencia a la cuestión de si los futuros traductores deben ser capaces de traducir únicamente al neerlandés, ya que sobre la importancia del idioma hubo consenso.

\section{Figura 1. Orientación al mercado laboral}

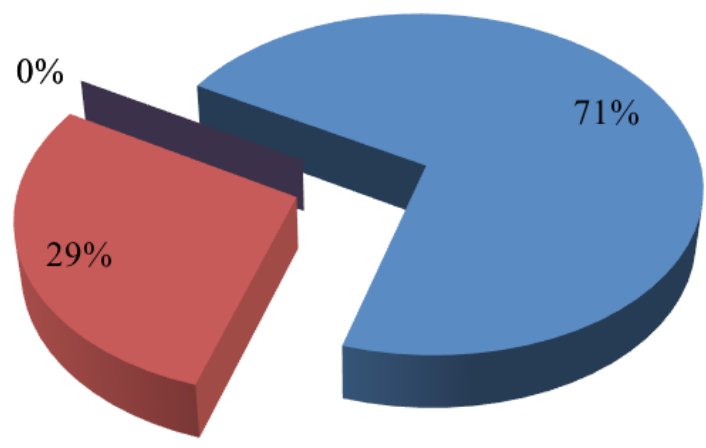

Esencial

- Útil

Secundario

- Innecesario 


\section{Figura 2. Bagaje cultural}

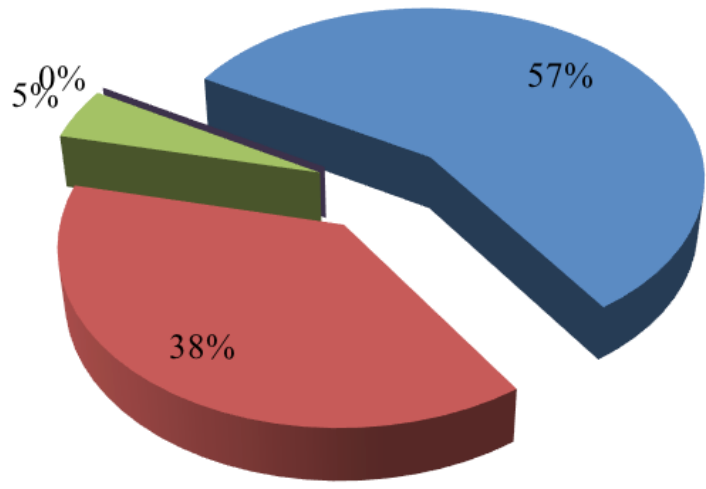

Esencial

घ Útil

- Secundario

- Innecesario

Figura 3. Neerlandés

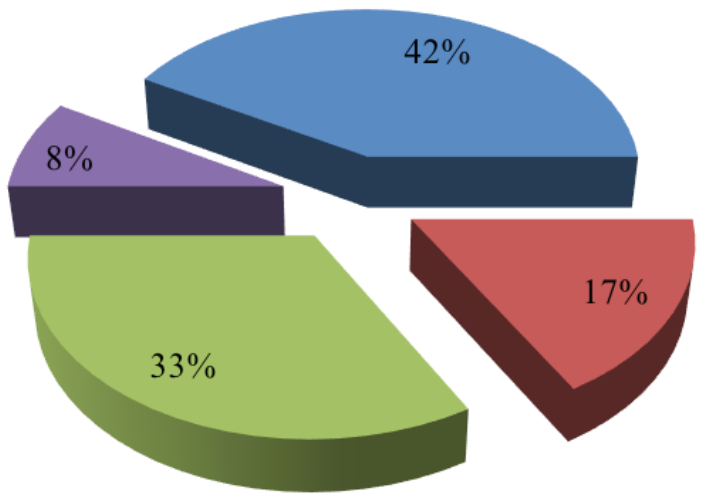

Esencial

- Útil

Secundario

Innecesario 
Figura 4. Lenguas extranjeras

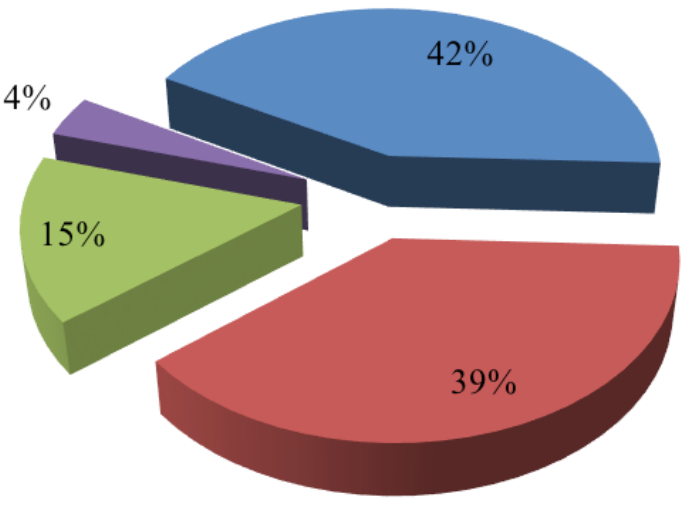

Esencial

- Útil

- Secundario

- Innecesario

Figura 5. Análisis de texto y estrategias de traducción

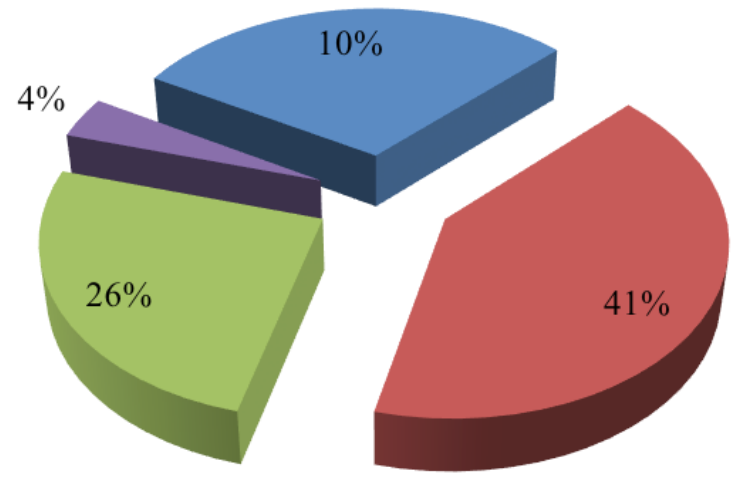

Esencial

- Útil

Secundario

- Innecesario 


\section{Figura 6. Competencias sociales}

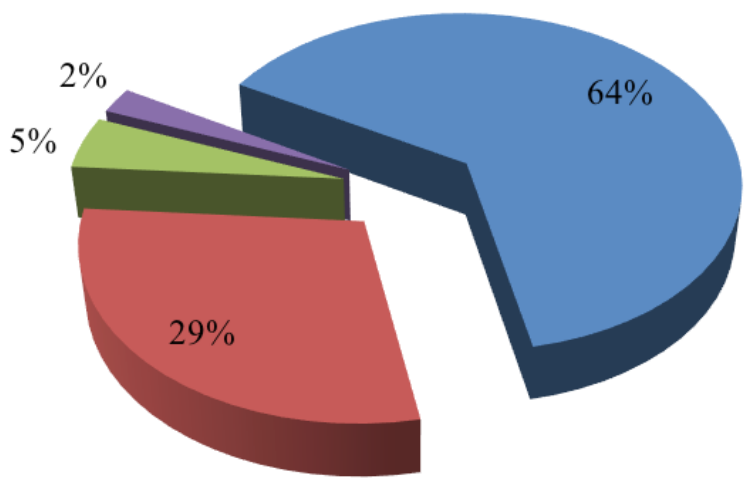

- Esencial

- Útil

- Secundario

- Innecesario

\section{Figura 7. Especialización}

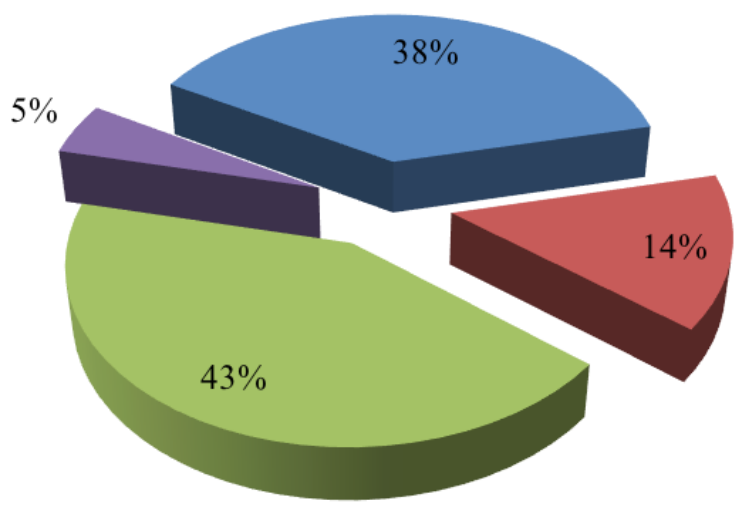

Esencial

- Útil

Secundario

- Innecesario 
Figura 8. Herramientas informáticas

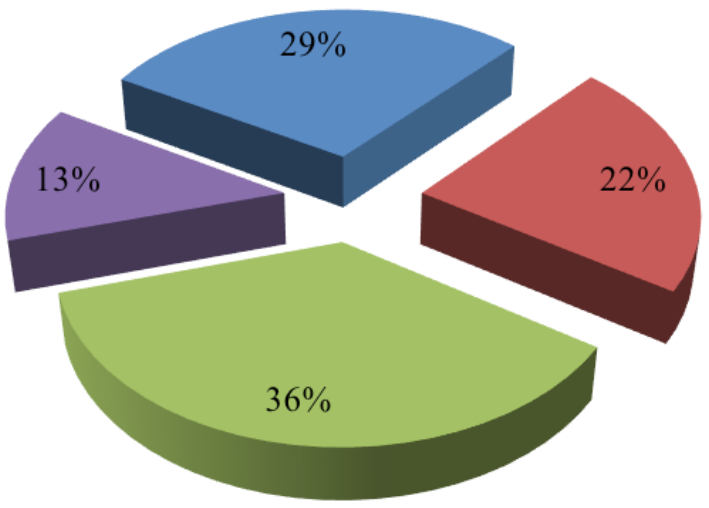

Esencial

- Útil

$\square$ Secundario

- Innecesario 